\title{
$\mathfrak{S} \mathfrak{D} \mathfrak{x} \mathfrak{t} \mathfrak{e} \mathfrak{d}$
}

Oolgende B̧lätter bebủrfen wohl bei ifyer Şernusgabe feiner Entiduldigung. Sie enthalten niche etwa blob Denfimáler einer gegenwártigen నeit, fie begiehen fi(d) nicht auf irgend ein Privat = Sntereffe ober auf irgend ein Berbàltniß einer augenblisflidjen Neuerung; fondern es fino Denfmáler Des bíchften, allgemeinften Intereffes für Die Bufunft, fủr Die $\mathfrak{A} u s b i l$ oung, Beridhtigung Des Bernunft = uno Criminaltedts. Die Eegenwart trágt oft nut zu felyr Die Epuren Der ßergangenlyeit, und es bedarf einer Infrage, of das, was yergangen uno gegenwártig ift, auch immer befteben foll, ob es yer = nunft= und ted tgemáf ift. Denn Die Erfafrung fpricht ja wohl laut genug dafúr, dak die Bildung ber Menfdbleit eine 2 lute ift, die nur yon bem unters ften Boden ftammt, fich nur nad) und nach in Dem Berlaufe yon Salorbunderten fu freieren Geftaltungen emporrid)tet, bis fie endid) uno zwar felye fpát die Srone Det Freitheit trágt, in welcher fidh Die Gejebe

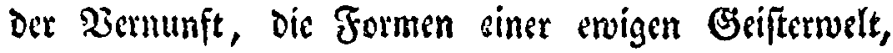


Durd) fichtbare Erfabeinungen Darlegen. Die (Sej(hidhte Der Menjhbeit ift, yon ibrer Raturjeite betrachtet, ein Sind Der Gewalt, eine Frudt Der Notbwendigfeit, ein Ergebnif Des bodbften Uebelftandes, wo Genult gegen Gerwalt fámpft und in ber irdijchen Mafle ber $\mathfrak{B e g e =}$ benheiten feine Rettung oder Berjölnung zu finden ift. Aber über Diejem Nothftand Des gejhidhtlichen Seins und Lebens thront eine holbere Madbt, - Die Madbe Der $\mathfrak{B e r n u n f t , ~ d i e ~ F r e i h e i t ~ i n t e r l i g i b l e r ~ B e f e ß s e ; ~ u n d ~ f o ~}$ befommit aud nad) biefer 2 nficht bie Gejdid)te sine hoblere Erleuchtung, fie mü endlich Der Bernunft Rede ftelsen, ilgre Gewaltthaten rechtfertigen und Das Wert Der frúberen Mlotlywendigkeit Durd) eine [pátere Freilyeit verfláren und fid zur $\mathfrak{B e r n u n f t ~ - ~ z u ~ v e r n u n f t g e m i ́ ~}$ Fen $\Re e d$ teanfid)ten erbeben. Der Stant ift nidht min= Der, wie eine jede andere irdifd)e (Erfd)einutig, iafser= lid) Das Berk Der Noth, die Gerwalt eines àuferen Zufalls, die J̦andlyabung finnlicher rober אräfte; aber in jeinem hobern und intelligibeln Wejen ift uno foll ex auch Das నeugnißß Der Dernunft fein, Die Deran=


Der Perjónlichteit, Der Freilbeit auf Eroen grúnden. Wáre Der Staat nidhts anderes, alỏ nur Der Reprå= fentant eines Figenwillens, Dem Die Gienalten Der ein= zeInen finnliden Naturen, Der Indiviouen úbergeben wåren: fo waire Durd) einen fold)en Gefammtwillen, wo Die allgemeine Gewalt gilt, nid)t viel gewonnen gegen jenen fogenannen Maturftand, wo die eingelinen Bewalten, Die eingelnen Actionen uno Rieactionen Das Edwerdt, Das (Fifen Der Jeerridafaft und Der ßergel= tung fưlyren. Der Sthut ift Die Madt Der $\mathfrak{B e r}=$ 
nunft, weldse auf Freiheit rubt, die Freiheit ausbrei= tet und befeftigt. Gie verjlinmált eine jede Gewalt= that, Denn fie ridhtet nad) Dem ewigen Richteriprudbe Der Perfonlid)feit und Freibeit. Fs ridhtet bier fein Sberer Den Niederen, fondern alle find gleid) und frei yor Dem Gejeese, und Das Gefeş erfennt in jedem, Dev yor ilym Sedbt fteht, ren Bater, Den Bruber, Den Soln.

Der ficherfte Maap̧fab für die Eultur eines Stan= teb oder $\mathfrak{R} a n d e s$, wie weit diefe gedielyen, wie felyr fie furutfgeblieben, ob fie fich von Den Uebelftanden des früberen Beiten gereinigt labe, iff befonders bas $\mathfrak{E}_{\mathrm{r}} \mathrm{i}=$ minal = oder $\sigma_{t r a f r e d t}$. (Es ift gleidhjam Der Mei= lenzeiger, in welchem Preije der $\mathfrak{B e r t l}$ des Menjdhen ftelyt, ob Der Stant nod) als aufere Bewalt, oder als freie Madht Der Bernunft ridite, ob er Das allgemeine Intereffe ber Siderheit, Der Rulbe, Des Wohlfinnoes, Der Perpónlidbeit, Der (Siered)tigfeit yon barten uno ůberlyarten Gtrafen, die wie mit flammenden Sdbwerds tern den Eingang des Paradiejes faúkęen follen, $\mathfrak{a} b=$ bingen lápt, oder ob ei einen bóberen $\mathfrak{R}$ ed t $\mathfrak{s}=$ grund Der Freibeit, Der Bernunft und Se= redtigfeit fid ju eigen gemad)t hat. Unter die Fragen, weldbe unjere fo beregte Beit angeregt lat,

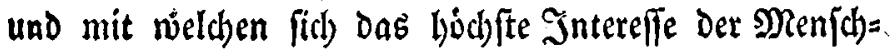
Leit yerbindet, nuf Deren Beantwortung entweder Das Alter einer alten tyrannifchen Beit, oder Das Şerbeia Eommen verjünter und aufgeflàrter Sabrhunderte be: rulst, - unter diefe Fragen, fagen wir, gehort audb mit Peebt die úber das $\mathfrak{R}$ ed t Der Tobegftrafe,

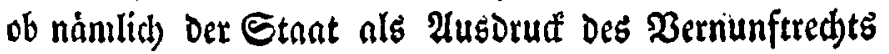


Die Madjt, ober vielmely die Gewalt fich anmafin Dúrfe, mit beiden Şánden gleidbjam Eroe und Şimmel zu umfaffen, oder diejen Hebergang mit Der Gdjneide Des Gdwerdtes ju lojen uno den Lebensfoden des Sterblichen früher zu zerreißen, als es felbft vielleidt)t



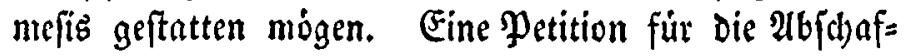
fung Der Todesftrafe ift nidjt etwa blos an ein sine jelnes $\mathfrak{B o l k}$, an ein einzelnes $\mathfrak{R} n$ nd, an eine befondere, eingelne Seit gerichtet. Denn fo viele Stimmen Der 3eit, Des 2llters, Der Senohnheit, einer gewiffen poli= tifden Nothwendigfeit fich audh wiber sine folche $\mathfrak{P}_{\mathfrak{l}}=$ tition erkeben mógen: Die Frnge und 2 Bitte behalt ifgre envige Gúltigfeit yor Dem Forum Der Dernunft, und frủber oder fpater werben und múffen bie Todesftrafen nbgeidafft werden, uno fünftige Generationen werden (iil) Dann einer gered)teren Iegislation, eincs milderen, vernunftgemáferen Strafrechts freuen. Die Sirunde, weldbe man fưr bie Beibelyaltung, ja fưr die Red)tmá: figfeit Der Sodesftrafe beizubringen pflegt, find man= Werlei $\mathfrak{A}$ rt und Uriprungs. Man modjte fagen, eine àltere DogmatiE Der Slbeologie babe ibre Şóllenftrafen in unferen Seiten befonders einem Dbjarantismus der Rechtsphilojophie und einem Myfticiomus bigotter Aergte anvertraut, fo da yon ewiger Erbjünde und א̧ollenftrafen tråumen und fúr Die Notlfwendigfeit, für Das $\mathfrak{B}$ erfólynungsmit= tel Des Scodgeridbts ftreiten. Die einen viffen nid)ts Sefferes füt die Todesftrafe zu fagen, als Dnf fie nach Dem Gefebce Der Iation und Reaction noth) wendig fei. So mogen auch jene Bilden philojopli= 
ren, bei denen Die Strafe des Scallpels, die Şaut von Dem Ropfe und Dem seibe zu ziel)en, naturrechtlid) eins gefülyt ift! Andere legen Das bleierne Material Des georudten $\mathfrak{B u c h f t a b e n s}$ zum Grunde, eine áltere $\mathfrak{E}_{\mathfrak{n}}=$ rolina habe die Todesftrafe einmal cingefút)rt, und $b a=$ bei núffe es yerbleiben. Diefe anderen pflegen gern diejen $\mathfrak{B u d}$ flaben Der (Sewolynlyeit ooer einer geniffen politijaten Nothwendigfeit noch) mit Redensarten yon vermeinten bóberen Beziebungen Der Ertenntniß ause jujhmútéen, ja ifjn wol)l gar Durdh Múllners Sabuld, over beffer nadb Den griedsifden Sragifern einer arifto. telifden Poetif zu erweifen. Ilber wer erfennt nidjt in foldhen Bründen, an einer fold)en Beweisfülyrung bie falichen Miotive einer aeitplilojophie, beren thoner= nes Jufisgeftell gar bald umftúrgen muß, um das ganfe Standbild mit fich herabzureifen. (Fine oritte $\mathfrak{A}$ rt von yermeinten Brunden fưt die Beibebaltung Der Todes= ftrafe ift die liebe faule Myftif, die yon Der Sobarfe

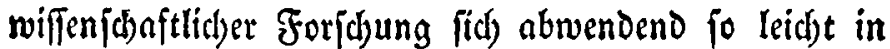

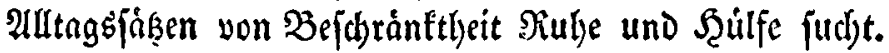
Es ift ein verdrięlides Sefdjáft, foldhen Siúnden zu


ben. Denn meiftens trifft man unter diejen Grúnden, weldje Das Ried)t Der Zodesftrafe erweifen follen, un= Iautere, unfiare Gedanten, falfhe Borberjâse, unrid)=

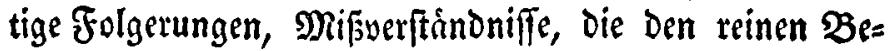
griff Der S3eredhtigfeit wie zum Sebel eines Medbanis= mus madben, und wo die Seredtigfeit Der Strafe nidht viel anders ift, als Der blinde Fall eines B̈iegels auf Das STaupt Des Shuldigen. 
I(ten Diejen Grủnden, bie mit po vieler Freimú= thigfeit gegen midh jprachen, habe idh mit gleicher Frei= mútbigfeit zu begegnen gejucht. Und fo glaube id midh in ber 2(trt und 2 Beife, wie die Begner mith) naljmen und wie ids fie nalim, Durd) Die Befege ge= genjeitigen $\mathfrak{B e r t r a u e n s}$ und gegenjeitiger $\mathfrak{B}$ illigteit yoll= fommen gered)tfertigt.

Es liegt am Tage, Dne die bister angenomme= nen Grunofáke oder Maçimen des Strnfrechts ferner nicht gúltig fein fónnen. Alle Die bisherigen Theorieen Des Criminalredbts leiden an Der Llnzulánglid)Eeit, an

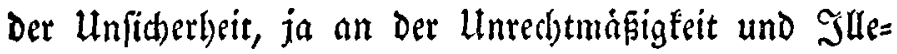
galitát ilyrer Yyrincipien. Das Strafred)t, weldes den Gepę̧en Det Bernunft gemàa fein foll, muß bie Frei= Geit, Die Perfónlidsfeit der Menfd)ennatur anertennen, - ein Ried)t, weldbes unyerleaglich und beilig ift. Uno Die Etrafe, weldye redtsts= und yernunftgemás fein foll, muß und Darf diefe Perjónlidseeit, diefe Freilheit Der Menjhernatur nid)t yerlezen, vielmely dieje, oder diefe Red)te felbft Dadurch in Der Etrnfe anertennen, Dấ fie nidbt gegen Das freie, intelligible Menfd)enleben,

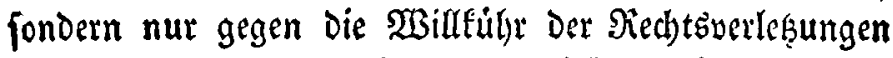
gerichtet ift, daßj fie, indem fie diefe coercirt, Der pers= fonlichen Freiheit Der Menfohennatur ifyre ewigen, un= verletb̧lid)en Recbte zugeftelet. Durd) Die Tudesftrafe wird Das envige Red)t Der perfónlidben Menf(bennatur beleidigt. Durd) die Bándigung der Willfứly des


fam wirb auf feine Beife, wie man wider midh eins

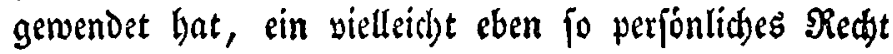

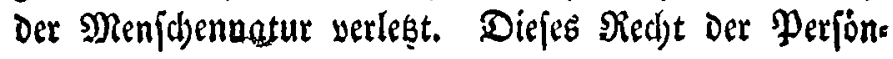


lichfeit wirb yielmelye, $D a \beta$ id) es fo ausbrúcte, intes gritt, in jeiner Sintegritát erlynlten. Denn die moralis idhe, perfönliche Exiffenz des Menj(d)en beftel)t ja nicht in einer $\mathfrak{B}$ Billfúlye zuffálliger $\mathfrak{B}$ ewegung, zufälliger Triebe, fondern in Der Unteroronung, in Dem 3 wonge Derfelben unter Den Gejegen aller jener humanen Er= weife, wodurd) Der yermunftgemáae Berfelyr des menfd)= lichen Revens erlyalten uno unterlyalten wird. $\mathfrak{D} \mathfrak{c r}$ in Der Zưcht Des Befängniffes, Der arrbeit, Der Strafe get)altene $\mathfrak{B e r b r e d}$ er iff uno niro nidht unfrei Durch Dieje Gtrafe; vielmehr niro und foll Die Bernunfthla $=$ tigfeit Der Freilyeit in ilym Durd) Die Etrafe, Die fidh


rid)tet, geweeft werden. So trígt aud Der Begriff Der Strafe Den Moment der Befferung als unmittel= bar immanentes Mertmal in (idd, weldes wohl yon Dem Zweftbegriff der Strafe, Der fo oft mit jenem immanenten Merfmale verwed) felt wird, unteríchieden werden muf. Die Strafe hat zwat mid)t Den Zुweff, zu beffern, aber fie trágt bie Mógliduteit, Die Betljáti= gung Der Befferung ummitteltoar in fich. Und nadt) eben diejen Eriterium unterfasetoen wir nun auch die gerechten yon Den ungerechten, Die milden und yernunft=

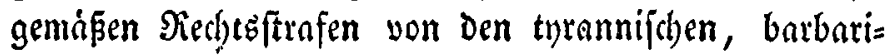
faten, blos finnlichen Rórperftrafen, die den Menjden

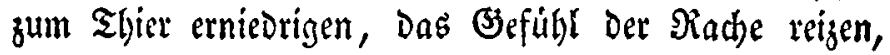
uno wo bie Annenoung eitrer foldhen Strafe unmóg= lich als eine verdiente, für Deren Ginnde fiith wohl gar nocb) Der $\mathfrak{B e r}$ bredber zu bedanten lyabe, zu nennen ift. Wir tadelm in Der bisherigen Strafgejesgebung nidjt etwa blos das eine Eapitel Der beibelyaltenen oder bei= 
jubehultenden Sobesftrafe, fondern bas innere seben uno $\mathfrak{W e j e n}$ jener Strafgepesgebung felbft, indem fie die Strafen bis zul einer jold)en 2 rt, $z^{u}$ eimem foldhen Grade fo oft auszudelinen pर्fegt, Dâj fie unred)tmáajig, jneffwidrig, Der Wúrde der Menfdennatur entgegen find. Die Etrafen ftrafen fith aber, múcte man fae gen, felbft Durd) Dieje ifre Excentricitàt und Şdrte. Denn Dicje Strafen verjchulden nun erft die Bösars tigfeit der menj(blichen Natur, die immer böswilliger Dutch Die erlittene ungeredte Strafe wird, wo der $\mathfrak{D e r s}$

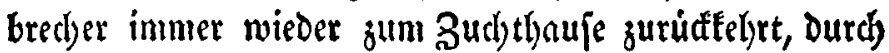
jenes Syftem ber Riorperftrafe immer prúgelfauler, und endlid) alles beffere (Befúbl Der Ifdtung Des Menjdhen fúr fith und andere herabgeferzt und gänjlich) erftift wird. Fragt man die Etrafgejesgebung, nad welchem Manfftabe fie fo und fo viel Saljre Suchthaus ver= orone: jo ift Diejer Manfftab rein willfúfyrlid), er be= rubt auf Dem sángenmanje Der frưlberen loarten Straf= fentenjen, wo die Milde und Biloung Der Seit faum fo viel vermod)t hat, einige Soll von jenem alten fdar= fen, harten Strafinfteme abunelymen. So lange in Der Sriminaljurisprudeng nid)t Der Srundjạ, Das ewige Gejeßs der MRenjdennatur (Fingang findet, Dâj geted)te und milde Strafen mely coerciten und corrigi= ren, alb barte psinlide Strafen: fo lange roftet nod) ein alter Fleff Der Barbarei in Den Strafgelesen, fo felyt aud dieje yon Den Rechtennwalden yertheidigt uno

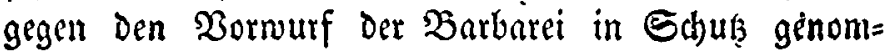
men werden mógen. Man fdlage Die Sabrbúdber frú= berer Strafgejeggebung auf und erftaume über den er= finderijosen Geift, mit weldsem die Gtrafen angedrobe 
und vollftrefft worden; Strofen, die in jeder Şimfidut Dic Múrde Der menfblid)en $\mathfrak{T a t u r}$ entel)rten uno ein Settgnif yon Det Robbeit, Der Unmenfwlid)feit eines unrecbllichen Strafinftems waren. Bon einem foldhen Bornurfe, Dev meldr als ju jelyr Durd) Die (Sefdidte beftátigt wird, wiro man Das frúbere Etraffy/tem nicht retten Fonnell. Man bringe einen Burger, Der von cinem civilifirten, gebildeten $\mathfrak{B e t n u n f t f t a n t e}$ Der neueren Sibelt geljort lyat, auf den offentlid)en Marft unfers Lebens, wo gerabe vielleid)t die jogenannten Exempel yon Strafe ftatuirt merden, und wird er nid)t erftaut= nen múffen ủber Das 2Bort yon Bildung und Gered)= tigfeit, weldhes man yorzugeneife neueren Sahrlhunder= ten beilegt? Nicht mit Unred)t dưrfte man ein jol= d)es Etrafuerfahren mit jenem alten Pennaliyftem auf Sdulen yergleiden, wo die Strenge Der Strafen Der SManjftab der Gultur und Der weifeften Paidagogit fein jollta. (Es thut uns leid, in Den lanoftándijaben Berfandlungen oft yon fold)em Exempel ftatuiren, yon der Siothwendigteit harter, abjhreffender Straf= mittel baben lejen zu miffen, als wenn es nicht audh boje Exempel gåbe, die man ftatuiren fơne, uno als

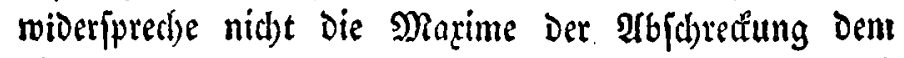
bohern Gejeşe Der Moral, unred)te Mittel nicht Durah jogenannte Swedfe zu heiligen. Es thut uns leio, wenn Die Rechtmápigfeit Der Todesftrafe Durch Den Ausfprud) ciner geniffen politifhen Rotlywendigfeit vertheidige

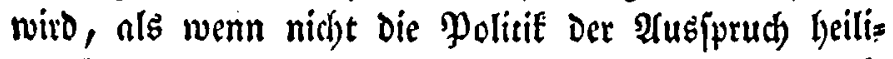
gex Bejesgebung und Der Gtant dic $\mathfrak{A n w e n d u n g}$ rei=

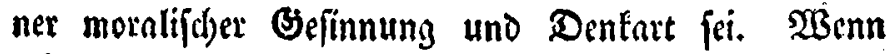

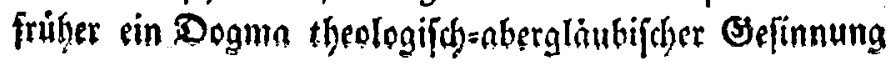


mit Redt yon ber weltlichen Surisprubenz Gelacht und yerppottet wurde, als feien ewige Silllenfrafen ber walure Erfał̧ Der Tugend und Bergeltung: fo lodert diejelbe Strafe in jenen aleen barbarijben Strafbeftim= mungen, Deren Blutfleffe jeb̧t nod) in Dem Solvwerde Des Şodjgeridhts eingegraben find. Der Stant, Der Den Jefeçen der Bernunft gemáp jein will, exlaube fich nid)t den $\mathfrak{B}$ egriff des 3 wanges zu úberjdereiten. Denn Diejer ift Die Brenge, Der Manjןtab der zu beftimmenden Gtrafen. Der Z̧wang ift nidt robe Naturgewalt, nicht fórperlidbes Sudtmittel, nid)t finnliches, entelyendes Strafúbel, fondern die Madbt Der Freilyeit Der Bernunft, Die fidh Durd) innere mora=

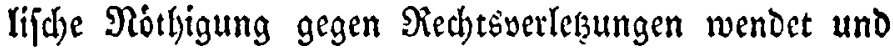

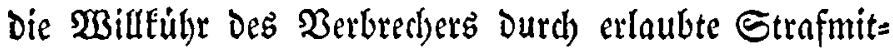
tel Der Bernunft zưhtiget uno zügelt. Dieje erlaub: ten Rechtsmittel Der Strafe fino námlich Correction Der Willfúly Durch Die Freilleit. Die Majeftàt Des Stants ift nid)t Raturgenalt nach irgend-einer Ma= fime medhanijber $\mathfrak{W i e d e r v e r g e l t u n g , ~ o d e r ~ I a t i o n ~ u n o ~}$ Sieaction, fondern Der Gobere Begriff, Die Miacht Der (Bered)tigfeit, Bernunftwe en audh nur Der Bernunft gemás unter Das Orejes Der Freikeit zu ftellen und die Perfonficheeit Der menfdlid)en Natur bis fum Srabe, ja uber Das Srab binnus gelten und beftehen zu las= fen. Fs ift nicht zu begreifen, was man fich unter Der Perfonlidfeit des Mienfdhen Denfen mag, wenn

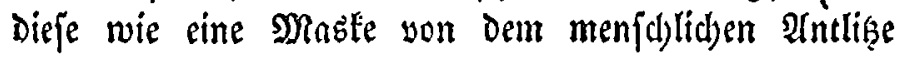
weggenommen und der $\mathfrak{B e r b r e c h e r ~ a u f ~ G a r i t e r h a u f e n ~}$ und א̧od)geridbt gebradjt werden fann, um als medba nifhes $\mathfrak{B e r f i g e u g}$ unter medbanifher Gtrafe zu leiden 
und zu - enden. Man beruft fith bei Der Bertheidi= gung Der Todesffrafe fo oft und am allermeiften auf Den Begriff Der Errechtigfeit, olyne Diejen Begriff ge= nauer erórtert, und Das, was medhanif de eimen redbten Winfel macht, yon Dem Segriffe Der vernunftgeniáäen (Sored)tigfeit, die perjonliden $\mathfrak{B e}$ en zufonmt, unterfbieden zu baben. Der Begriff jener to oft altb= gefprod)enen Gered)tigfeit ift ein fenjueller, er pafat nui auf Das, was nadh Ellen gemeffen und beftimmt werden fann; er ift nicht Der intelligible Begriff, Der Die unverleb̧lidbe $\mathfrak{B e r n u n f t}$ fum Grunde legt uno nid)t Die $2(d)$ tung yor Dem beiligen Eefese der Perfönlid)= feit yerleşt. Der walunfinnige Buftand Der Seele, Der in Den Srrenlyåujern Der Şeilung Des Irgtes ủber= geben wird, entlyebt ans nicht Der 2ddutung, mit wel= d)er bie perionlid)e $\mathfrak{R a t u r}$ des $\mathfrak{M}$ alyminnigen belyandelt werden foll. Die Perfonlicheit ift Der úberfinnlid)e, úberall vermelynliche ruf Dev Bseifterwelt. Eben fo wenig ửberbebt Daber aud) Der $\mathfrak{B a b}$ (inn irgeno eines Berbred)ens Das Strafant, in Dem Berbrecher Das perfonlidje $\mathfrak{W e j e n}$ nicht gu achten und ilyn auf ben

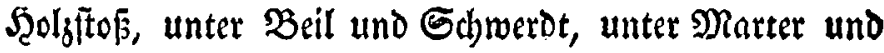
Gtoff zu bringen, um den Gejeşen einer roben medbanijoben Betedhtigleit gu entjpredben.

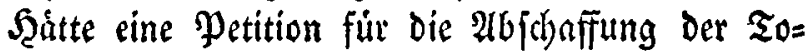
desftrafe Den gưnftigften Fingang gel)abt und die $\mathfrak{T}_{0}=$ Desftrofe wáre einftimmig abgefdofft worden: was bátte man fừ Ultheile bören múffen, wenn unmittelbar nach foldber $2(b j$ djoffung fich) Berbredben gezeigt båtten, die jonft gewóbulich mit dem sobe beftreft zu werden pflegen und jeşt nun einer gered)teren und vernunft= 
genióperen Beftrnfung anleim Ifielen? WBüde man nid)t die 2(bj)affung Der Todesftrafe fúr Das fdrei= endfte Unred)t, und jente Blutfdulden fuit Erfolge

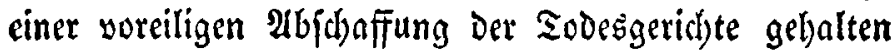
Gaben? Die Erfalyung lebrt, Das Durdh barte Stra= fen, Durd) Sodesgerid)te Berbredjen nidbt gemindert werden. Aber fo lelyet freilid) auch die Bernunft und

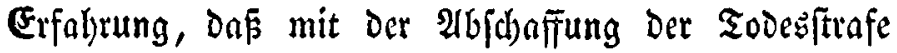


fobwinden werde. Denn bei aller Eultur des Men= fdengefdledbta Eeimen Dod immer Lafter, Leidenjofaf= ten, betwuftlofe Suftánde, welche ju Sdjuld uno $\mathfrak{B e t}=$ bred)en Anlafa geben. SBei allex glüctichen Srganifa= tion Der Menjdhennatur giebt es fo viele Abweid)un= gen, wo Der Menfch melye Den sliere als Dem Sciml= mel náber ift, und wo die abweichende Dignnifation nud) Der Seim uno ber ßoden Des Berbred)eris ift. So wenig Blutgerichte $\mathfrak{A b}$ (d)reffungsmittel yor $\mathfrak{B e r}=$ breben find, eben fo wenig wird freilid) auch bie $\mathfrak{A} b=$ fdhaffung Der Todesftrafe die fermeren Erjcheinungen blutiger $\mathfrak{B e r b r e d}$ en zu unterorúcten vermógen. Denn, wie gejagt, auf allen verjhiedenen Graden Der menjth= Iidjen Cultur bleibt die Smbecillitát, Der Sirantletits= ftoff finnlid)er ßrafte fidh immer mehr oder weniger gleich. Alber wenn auch jold)e Berbrect)en und Miffe= thaten aus Dent Reidje Der Menjableit nicht ganz yer= fd)winden fonnen, wie man auch die Strafgejese ein= ridfte und anorone: fo ift und bleibt es Dod Der hei= ligfte Beruf des Strnffyftems, fich als ein geredtes, yernunftgemáfés Syftem zu erweijen und nidbt Durch unred)tmisfige, vernunftwidrige Strnfen bie Rind)e des 
Berbreche: bredben auszufâen. Die fidberften, die beften Mittel, nidjt bij $\{e$, fondern $g$ ute Exempel zu ftatuiren, find für Die Regislation Die (Frziebungs = und Buchtnittel, Die in Lefre und Unterticht, in Ausbreitung uno $\mathfrak{B e r}=$ edelung Der Edulen, in Erridtung yon Befferungs= Şåujern beftelyen, wo melye moralijdbe, als finnliche oder forperliche Wilffommen und Strafmittel ange= wendet werden múffen, um Die Freilheit yor Rechtver= Ieşungen zu fid ern. Wie viel bleibt aber zur (Errid)=

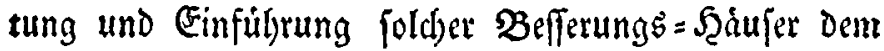
Stnate fu thum übrig! Slur erft in ben neueren $3 e i=$ ten lichtete ein $\mathfrak{h}_{0}$ watd die Dunflen, fobredfliclen (je= fängniffe, in weldyen die Berbredber allen Elementen einer feindfeligen Natur preisgegeben waten. Die Derbeed)er, wie fie den Dunflen $\mathfrak{S}$ b̧blen úbergeben wut= Den, waren gleidjian ausgeftofiene, vergeffene Blieder Der Menjd)heit: Rur erf in Der neueren Beit wanote

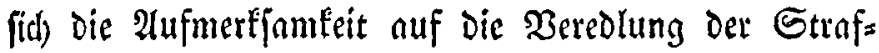
gepergebung, nicbs Durd) Tortur, Durdh Infinuationen, Durd) unmoralifdse Mittel, Durd gebrime Suftiz bar= ter Belsandhng Berbredben zu entoeften, Seftand: nilfe - auch falfde und ergwungene, wenn der ans

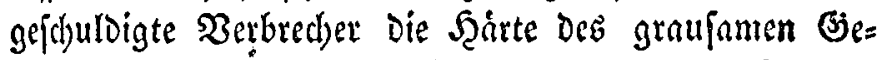
fángniffes niclst ertragen fonnte - zu erpreffen! Befegnet feien Die Saljrlyunderte und Deren SBemúlyun= gen, Diefc Giratul der Strafjuftiz nbjuwenden und úberall cinem yernunftgemáperen, milderen Syftente, die Balbin zu brechen. Alber fo grof audb diejer Se= gen Der Sallrhunderte und ilfrer Bemúlgungen für Die gefalleme Menfobleit fein mag, fo ift bod) nid)t in $\mathfrak{A b}=$






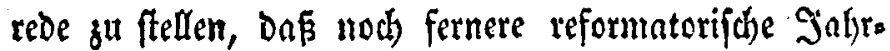
bunderte beyorftehn, welche bie alten Ueberrefte Der Barbareien in Den neueren Entwurfen Der Strafgez feşgebung zu tilgen und angzulojiden baben.

Bis auf bie neueften Beiten war nod) hie uno Da Das fogenannte Şalsgericht úblidh), wo úber den Berbrecher, ehe er zum ḩod)geridht geführt wutue, Durd) gräfliche fumbolifdhe Beidhen Der Stab gebrod)en wurbe. Weldhes sruftzeug yon moralijd)er Pein uno Marter erbielt nid)t fobon diejer einzige $\mathfrak{A}$ (ct Der jonft fo gexúfymten loodnotlipeinlid)en Beridhts = Dronung!

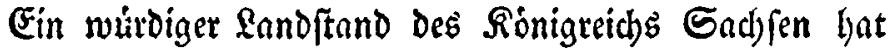
(iid) am Gd)luffe Der landftándijđben Berlandlungen ungemein verdient um Das $\mathfrak{B a t e r l a n d}$ gemad)t, die $\mathfrak{A} \mathfrak{b}_{2}$

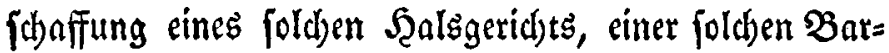
barei aus frubleren finftern Baiten zur Propofition zu bringen. Uno die gered)te, menjdenfreundiche Peti= tion wurbe yon ben lodigead)teten lanoftánoen, wie Der leşte $\mathfrak{N a d}$ )trag folgender Blàtter zeigt, wenn nidut ganz, Dod) Dem widhtigften und wejentlidbjten Jnlyalte nach einftimmig genelgmigt. $\mathfrak{D a s}$ Şalsgeridht if nun freilich abgeidafft, aber Der (Sang Dest Berbred)ers zum Şodsgeridjte ift geblieben; und wenn audi Diejer offentlide Esang, dieje iffentlidec, feierlid)e Sdyauftels lung des $\mathfrak{H}$ odhgerid)ts yermieden voer abgejdafft wúrde, bliebe Dann nidht immer nod) bei Der Bermeidung Dies fer Kleinen Barbateien die grósere und grópte, Die So. Desftrafe jelbft, fie mag nun iffentlich oder àuf eine weniger offentliche und feiertiche Beife erecutirt wer. Den. Der groffte Uebelfand bliebe immer bei allet Dermeioung Diejer fleineren Barbareien und graulac 


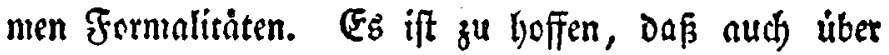
die Todesffrafe, weldse den leşten Streid) úber ein gefallenes Reben vollführt, enolid auch ein lester Streich Durd) Die Madbt Der bóberen Bseredhtigfeit und sines bobern Einflangs ber $\mathfrak{B}$ ernunft mit fid felbft werde volftrect werden. Denn bei allem ablebnens

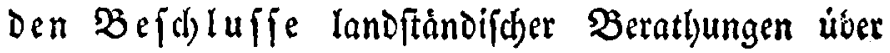

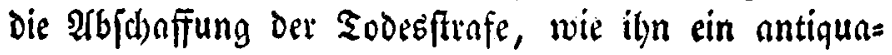
rifber Berichterftatter in ber allgemeinen 3 ei= tung nennt, lyaben Dod̨ die weifen lanoftàndifden Derbandlungen meines $\mathfrak{B a t e r l a n d e s}$ die von mir eins gereid)te Petition nicht verworfen, nidbt etwa zu Funf: tig zu revidirenden $\mathfrak{A} c t e n$ verwiejen, fondern um Der weifen, umfid)tigen Beratbung fallof willen, wie es Das lyeilige Intereffe der Menjdheit mit fid bringt,

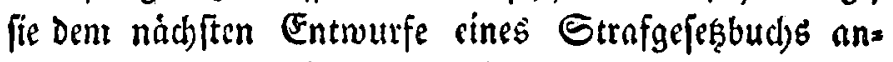
yertraut, weld)es úber die Billoung, ỉber die Cultur fommendev Solbrbunderte fúr die Strafrechtspflege zu enticheiden bat, Und wir geben uns gern Der troften= Den Soffinung liin, Daß ủberall ein mildereb, billigeres Etrafinftem Eingang finden, jene tragijd)en Mittel einer iffentlid)en Blutrad)e, Blut Durd) Blut zu fob: nen, nus dem Sande der mornlifden, religiojen und wiffenjd)aftlidben Aufflórung ver [d)winden werden. Bei Belegenbeit jener Iandftàndifden Propofitionen fủr die


folgender Manân über jein Slaubensbetenntnis, wie nothwendig die sodesfrafe fei, yernebmen.

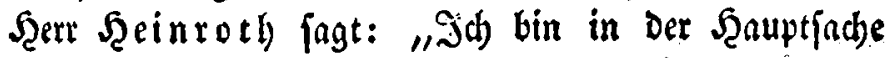
"gang Der 2 (njta)t Der geeljrten Deputation und eben "fo in Dar Şauptjad)e gegen die Anfid)t Des geefrten 


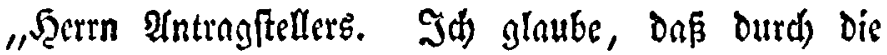

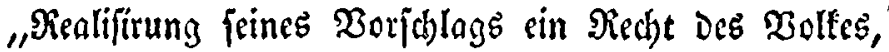
„nåmlich die Deffentlich)eit Der Todesffrafe, verleșt wird; "ein Ried)t zwom nid)t von Der pofitiven oder jecund $\dot{a}=$ "ren, aber yon Der primitiven $\mathfrak{A t t}$, ein allgemeines

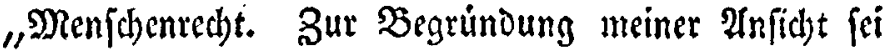
"mir erlaubt, Gier cinen 3 lif alf die gobe Wuárde "DeE Stantes zu werfen, ich meine námlidh die Wúrde, "weldye Dadurch begruindet wird, Daß Der Gtant nidst „eine Mafdine, fondern eine moralifase Merfon ift. "Es handelt fith int Etaate ưberhaupt nur um die "Perfönlibjfect. Diejer $\mathfrak{B}$ egriffi Durdboringt alle $\mathfrak{B}$ ers "lialtriffe und Begieljungen Des Gtants. Fr beftimmt



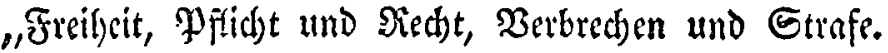
"Die Strafe iff nidtes Barbarifhes, fondern etwas

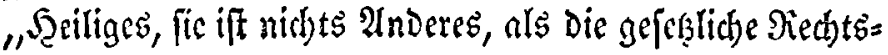
"verfitumerung gegen Riechtšverleşung. Nomentlich

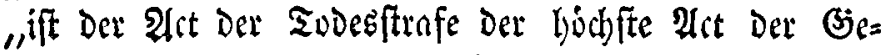
"rechtigfeit, welche Dab Princip und dic Geele Des

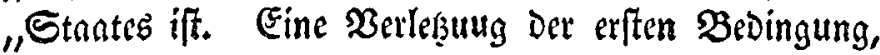
"unter welds)er alleint Die Perjon eqriftiten fann, ein


"Dourdi) Die Todesffrafe nusgeglichen, eine Sabuld gegen "Dns Seben nui mit Denz Reben gebúfet werdent. S̃n

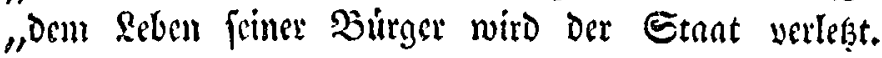

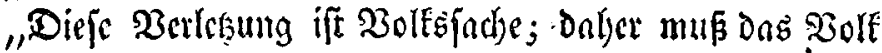

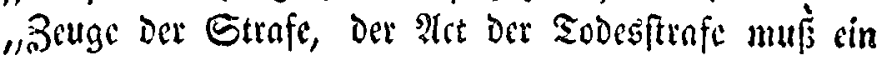
"ofifentlicher frint. STlm die Deffentlidsfeit rauben, ift

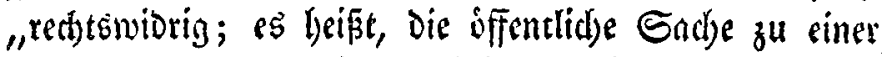
"Privatfadbe zu madben. WBiefern bei dem iffentlichen 
"Strafact die WBirfung ouf Die Gemuther berúaffich)= "tigt wiro, fo ift dicfe gewis in taujend und aber "taujend Geelen wolylthátig, wenn aud nidht immer "fid)tbar; und die wenigen Beifpiele, Daf Perjonen,

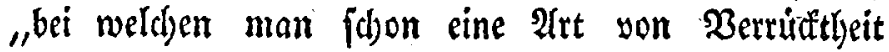
„,prijumitren mu仿, fich Durch sine offentlich yollzogene „Iodesftrnfe zu $\mathfrak{B e r b r e d b e n ~ b a b e n ~ y e r l e i t e n ~ l a f f e n , ~ F o m = ~}$ "men bier nidht in $\mathfrak{B e t r a d f t . ~}$ Bet der Eintidtung "Deb Strafacts, weldye Der geehrte Intragfteller vor=


"Denm Richter uno Sdyoppen und alle bie úbrigen "ahthaten Beugen Des Strafacts bedúrfen Dod) wohl

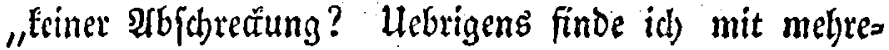
"ren geelyrten Spredhern vor mir in Der Einridjtung "unjrer Borfalyen, abgejehen yon Den Epuren Der „Sarbarei Des MMittelalters, etwas Einoringliches, Erm. „ftes, Seligiópes. Alth) sine Straferidyerung fann ids „in Der Seffentlichfeit Des Strafacts nicht finden, benn

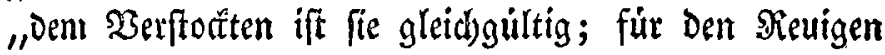
"aber ift es berubigend und troftend, Andere Durch 1) fein $\mathfrak{B}$ eifpiel yor Der $\mathfrak{B}$ ahn Des $\mathfrak{B e r b r e d}$ ens zu wars "nen. Dem siffentlichen Unfuge fann ourd) polizeilidge "Manäregeln gefteuert werden. - Sin diefem Stüce "muß id) aber Denl geelsten Antragfteller beiftimmen, "Dak Der Tag Der Jinridtung als ein Tag Der Trauer "bezeichnet werde, wiefern Die (Sejellidhaft genothigt "wird, eine lebende Geele yon fid) augzuld)eiden. "Jierourd) widerfályrt nidjt Dem Berbred)er Egre, fon= "Dern nur Dem Menjden 2Cnerfennung. Sib wieder=

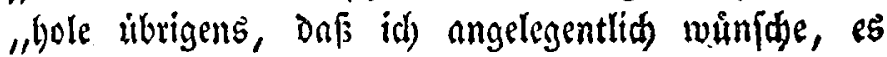


„mige yom iffentlichen Gtrafacte alle Epus von $\mathfrak{B a t}=$ "barei entfernt bleiben."

Sătte Doch, fabren wir ljer im Seifte des aud) von uns fo bod gead)teten Intragftellers fort, die $\mathfrak{P}_{e}=$ tition niche blos um abjonffung eines barbarifben Strang = und Standred)ts, fondern aud) unt eine vol= lige Ibolition der Todesfitrafe gebeten; es wurde in Der gangen Berhandlung eine Ber (b)iedenheit der Mei= nungen un Rleinigieiten, um Abjhaffung Flénerex Doer geringerer Barbareien yermieden worden fein. Denn leiber finden wir in unjeren neueren juriftif(t)en Unterfudungen eine foldbe zwar an fid nidjt tadel= bonfte, aber boh eben nidht wichtige Mifrologie ber Sewiffenlyaftigfeit, wie Der Berbrecher hinjurichten fei, ob auf einem iffentlichen Şodhgerichte, oder in einer Belime yon Sdhranten, ob Das Beil, Das Shwerdt, oder Dab Fafleifen Der Grifllotine sie Shintidstung voll=


yermieden, der Menfd) nicht blos als Thier oder $\Xi_{\text {adje }}$ bilhandelt uno Das Reben auf Dic kủigefte und fbos nendfte Weife verniclytet werde. WBir elyen zwar sine fold)e juridifbe Mifrolugie der Moral, aber nur nidt mit llngelyung Der Şaukfadse uno in folden Fâllen, wo Die Frage eime ganz andere ricijtung und boblere Bedeutung erbålt, ob nomlid die Todesftrafe mit den allgemeinen uno nothwendigen Oefetsen oer Moral, mit Den geláuterten Unfíd)ten Der dbriftlichen Religion,

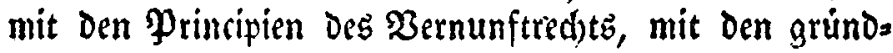


Jorjdung - furz mit Der 2ldhtung gegen die Per= jonlichteit Der Menja)ennacur und Dem enig unverlese 
lirben Sied)te Des menjolichen Geiftes! ưbereinftimme.

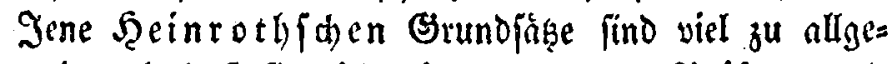
mein, als dâj fie nicht einer genaueren Pluáfung uno Berichtigung bedưtften. Sceintoth get) yon Den allgemeinften Principien bes $\mathfrak{B e r f t a n d e s}$ aus, die Sei= ner láugnet, ziehet nber aus den vagen Borderjăksen Folgerungen, Dic zwar einen philojoplifiten Edbein


nid)t befteben. Was beift es Denn - um nur (Ei= niges aus jener giede anzufúbren, - die Strofe ift nichts $\mathfrak{B a r b a r i j d ) e , ~ f o n d e r n ~ e t w a s ~} \mathfrak{T}^{2}$ eiliges? Wer mag diejes nid)t Dem Reoner gern und willig fuges freben, wenn wir auds bier mand)en (Finfpruch úber ons W̉ort Des Seilligen einjureichen bàtten. Folgt Denn aus Der allgemeinen Prániffe Der Sceiligfeit aud)

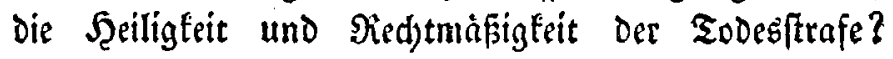


Ridjter, wenn er Den Sunder Durd) Strafe beffert, aber - man yerzeilye uns bie Folgerung - ein bodfft unbeiliger Siddter, wenn er wie ein menfálid)er (S)e felsgeber den Berbredber zu Tode bringt. Eine foldbe Strafe ift jwar die leidstefte und geidhwindefte. Man braud)t nut zu yernid)ten, was man gefdaffen, oder - nidft gejd)nffen lyat. WBas foll ferner jene Şeins




wenn auth in minderam Brade, eine Rechtenerleşung, und fo ftelst nun die Red)tsuerleşung Der Redbt= yerleşung wie zwei feindliche Nad)barn in Den Me: chanismus det Blutradi) einnoer gegenůber, eine

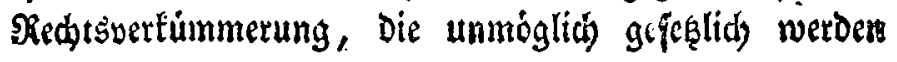


fann, wenn nid)t linted)t zum Reche werden foll. Seine Strafe Darf ingend ein perfönlidbes Red)t Des

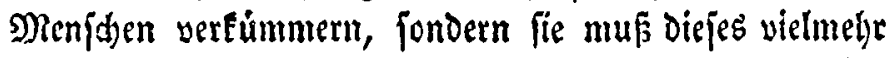
antertennen und Durdh ifgre Erfenntnif ausfpred)en. Der $\mathfrak{B e r b r e d}$ er, indem er geftraft wird, Darf nidt in


erhaleen, und ifm gegen die yon ifm begangene Rechtss yerleß̧ung zum Ģebraudbe Der Freitgeit, zur Mündigfeit


Berlesende in Den bisterizen Begrifen und Ifyeorieen Der Strafe, Daf lie nad) un[erer Hebergeugung Hnred)t

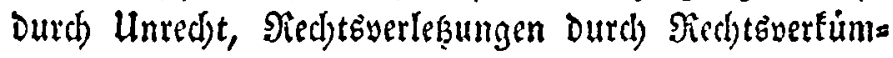
merungen gut zu nuaben meinen. Der Sieoner ger)t yon Dem lóblidben, unjroifellyaften Grunofas aus, Dee Gtant jei feine Mafdhine, fondern eine moralifde ঐer= fon. Tlber eben Darum fagen und folgern wir, Der Stant mus fidd nidjt als Mafdine brgeigen, indent er Den Berbred)er ale Majdine beljandelt, fondern die


Der Menfonenwurde elyen. Der Menfid barf nidit, und wenn er aud) der grofite Berbrecher iff, unter Dem Rinde gequet $\{d t$, burd) ben Strang eroroffelt, von Dem Schwerote gefopft werden. Der reoner nennt Die Serechtigfeit Das phrincip und dic Geele Des Gtants, olyne genauere Rechenichaft yon jenem Begrife ju ge= ben; Denn es giebt $j$ aud cine medhanifdhe Eevoalt Der (Stered)tigfeit, weld)e aber jener drifflidbe 2lusfpruch - "rid)t Zahn um Zn!hn, nidjt 2uge um Iuge" -





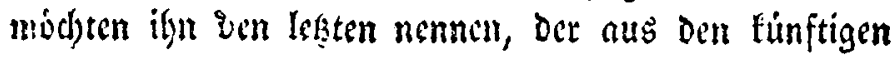


Entwüren befferer Strafgeféggebung ju entfernen ift. Dods unjere $2(n j i d) t e n$ fino zu jelyr yon Denen, weld)e obige Siede entwiffelt, ver[dbieden, als daß̄ bier ein ge= genfritiges $\mathfrak{B e r f t a n o n i ß ~ z u ~ h o f f e n ~ i f . ~ W i r ~ b e g e g n e n ~}$ Diefem ganzen Sceinroth'fd)en GHInubensbefenntmis

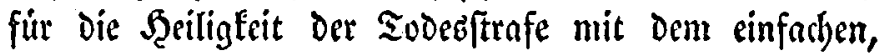
nber walyen Gaß̧e Der Moral Des gechrten Intrage ftellers, Serrn w. Miltib: "Die d)riftlidse Moral "lebrt ung, onf wir Dab $\mathfrak{B}$ bje nidbt uill Der Strafe "Ivillen, fondern Darum fdenen follen, weil ė bófe „ift. Sn Setreff beffen, was Der geefyte Sprecher "hinfidbtlich Des (Eindrutfs, weld)en eine Şimrichtung "auf Das $\mathfrak{B o l f}$ mache, crwallnte, lyabe idh zu bemer=

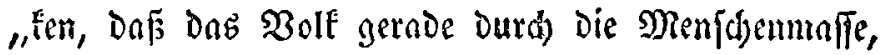
"weld)e fich gavobnlid) bei Şinridstungen einfindet, "nidht wohl yertreten werden tann, uno mache bepon= "Ders nuf Den ungewoilynlidben, Enum ju bejd)ieibenden "Sumult aufmerffam, Der yor und nad) einer Shin= "richtung fratt zu finden piflegt." - Soir Feken binzu, Dafis wir zu einer inconmenjurablen Serbmung des $U_{n}=$ enolichen fommen, wenn die unfid)tbaren woljithatigen Folgen, welche eine J̧imidhtung auf taufeno und nber tnujeno Seclen baben foll, gegen die fdlimmen, nber fidtbaren Folgen, Die eine Sintid)tang auf, wie heintotl meint, fhon balb walnnfinnige demuther bat, abgewogen, uno fo ein inconmenfurables $\mathcal{H}_{\text {eil }}$ Der Toderftrafe gegen Das Unted)t Derfelben in Betradt gezogern werden foll.

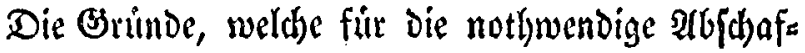
fung der Sodesftrafe [pred)en, find crftlich pind)ologi= fde, jweitene juriofidse, orittens moraliflye ooer reli= 
gioje und endlid anthropologifole, welche Die Erfah)= rung und Die genauere Beobad)tung Der Erfolge, die Gtrafvollftrectungen auf Die Gemủther herborbringen, an die Sand giebt. Die Pindologie lebrt, wie fd)wer Jandlungen Der Freilbeit oder bes jogenannten $\mathfrak{Y}$ or fậes und der 2(bficht von Den Şandlungen Der Notly wendigfeit, yon Den Ergebniffen eines Dunflen, vernol= renen, franfen $\mathfrak{B e w u f t j e i n s ~ z u ~ u n t e r f b e i d e n ~ f i m b . ~ D a s ~}$ anatomijthe Meffer des (Serichtsargtes und Pindbologen iff nidht fo fein, um dieje Fiden zu zerlegen oder ju trennen. Serfduneiden fann es dieje Fáden wobl, und Dies ift leider die empirifhe Runft vernlteter gerid)ts= àrtlicher Erfenntniffe. Die áltere Pindjologie und ges richtliche Seelenfunde blieb nur bei dem äufern Soma Der Erfdueinung ftehen, ofyne die tieferen Beheimniffe Des Seelennejens zu ergrúnden; fie manjen, um bier ein Seifpiel zu brauchen, Gecle uno Siorper nah weit gejeşten Srenzen ab, dhne auf die Slacbbarftanten ju jelsen, die zwifhen Seele und Rörper liegen und Das ganje Gtantsleben des Menithen beoingen. Fin frús


uno gerichtlidhe Erfenntnis trugen fein sebenten, auf Seufel und Seufalebejoboirung zu ertenten. Dutdh die neueren Unterjuduungen Der Seelenfunde ift nidbt allein langft Diefer grobe, fraffe Ifberglaube, Der $\supset \mathfrak{b}=$


find aud) bie feineren gelseimen Fáben aufgefunden wor= Den, Die einen gejelligen $\mathfrak{B e r e i n}$ von Sranfyeiten Des Geiftes uno Rorpers ftiften, uno wo Der $\mathfrak{W a k n f i n n}$ Des Srrenden fo oft Der $\mathfrak{B a}$ ahnfinn Des Berbred)ers, und 
Die Berfouldung Des $\mathfrak{B e r b r e c h e r s ~ f o ~ o f t ~ n u t ~ D e r ~ w a h n = ~}$ finnige 3uftand einer bewuftlofen Seele ift. WBns es mit foldben Unterjuchungen oer P(n)blogie uno einer Gelleren gerichtsågtlichen Sunde fúr $\mathfrak{B}$ ewnnonis babe, Daruber geben Die Beitfdriften, die Berfe cines $\mathfrak{\text { affe, }}$ Friedreith, J̧orn Die genúgendfte Belehrung. Eg ift nicht moglids, Daßs wir bei allgemeinen Berlyandlun= gen fút die $\mathfrak{A b j}$ daffung Der Todesftrnfe wiederlyolt in fo viel cingelne Unterfuchungen einer grüblidberen $\mathfrak{P}[\mathfrak{y}=$ d)ologie, einer genaueren gerichtẹirgtlid)en Foridung singeben tounen; alle bieje Unierfudjungen liegen als Borarbeiten in jenen Sdyriften der yerdienftuoluten Mản= ner, welden fich die Namen eines Groos, Bird, Almelung u. \%. w. beigefellen, in ber Itt uno Weife ju Tage, $D a ß \beta$ es nicht melyr $\{$ d)wer ift, ein entfchiede= nes Urtheil tiber Das Berbàngnis Der Todesjtrafe ju


ben auf feine Weife p[ychologif(h) und gerid)tëárgtlich in irgend eimem Folle erwiefen ober begrundet werden fann. Wir verweifen, wenn $\mathfrak{s}$ uns erlaubt ifr, yon unjern eigenen geringen unbedeutenden Beitrigen zu fpreden, auf die oben erwábnten Beit\}ádriften, wo wir glauben, mebrere Bemertungen und Beobadjtungen beis gebrad)t ju baben, weldbe die Gefaldr Der gerichtsárgt=

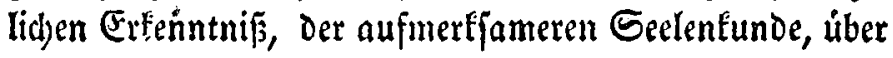
Sduld und Buredhnung, uber Freilyeit und Strafe zu urtheilen, in ein beftimmteres und helleres sid)t [eşen. Mit Den \{harffinnigen Unterjud(ungen, weld)e obenge= nannte fdarffinnige Männer in iljen Gdjriften nieder= gelegt haben, find zu yerbinden bejonders auch die phie 


\section{- Exvir}

lojeplifhen Erorterungen eines Gitoos uno Mel = ring úber das Innere des fo zarten Gewebes des Geelenlebens, wo die philofophifde Erfundigung fo viele Şweifel bebt, welche das anatomijáe Meffer nicht zerlegen Fann, uno fo viele Şweifel uno gerectite $\mathfrak{B} \mathfrak{Q}=$ Denflichfeiten findet, Die das anatomijhe und gerid)ts= argtliche Meffer oft allfu foryarf und allzu grob zerjonei= Det. Der auf feinem Tribunal thronende weltfid)e Rid =

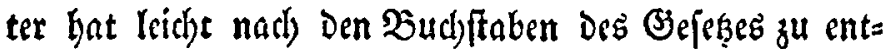
\{feiben, ilym jdbweben blos die alfgemeinen sermine yon Culpa uno Exculpation yor, or abnet nidft die Warnungen und Malunungen ber gelyeimeren $\mathfrak{B i f f e n =}$

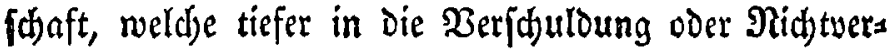
jhuldung Der Geele eindringt. Preisfragen úber pinchologifo Momente, in wiefern fie Die Unzulaffigs feit Der Tadesftrafe Darthun follen, wúrden zu nidjts belfen und Die Entidheioung Der Frage nidjt fordern. Denn Die Preisfragen wúrden immer nur neue Preib= fragen móglid und nothig mad)en. Uno, wie gejagt, Der pingologifhen Beantwortungen fino in Den oben genannten $\mathfrak{B}$ erfen fobon binreidyend vorlyanden, um jes Den neuen Alufwand yon Preisfragen unnothig und unratbjam zu madben. Gold)er y[ydjologifdyen Unter= fuchungen fonnte und wollte id freilich nicht in mei= nen fleineren Sdyriften, die id über das Gtrnfrecht herauggab, und nod) weniget in jener an eine land=

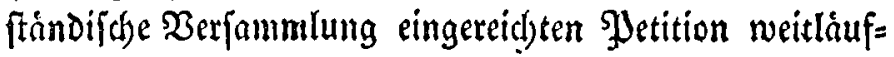
tig erwálinen, id) muste yorausfeşen, was befannt mar, und in biejen Edyriften úber Das Strafred)t und úber Das Unredbt Der Sodesftrafe war ja aud) nue die ju= 


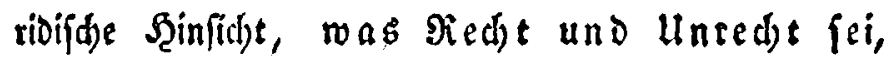
Frnge und Sade der Entidjeidung.

Wab die juribifden Principien betrift, nah wel= (hen für oder wider die आbjめaffung Der Todesftrafe, fúr oder wider bie Strenge einer alten und neuen Strafgejeggebung erfannt werden mus, fo beftelen Diefe nach meiner Uebergeugung in folgenden wenigen

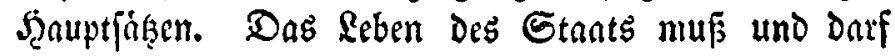

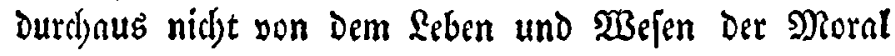
getrennt werden. Die Bernunft ift nur Eine, eine uno die námlidbe untheillbare Sottheit, Die in allen Eradei= nungen menjulidien Geins und Ḩandelns, in Firdse, Stnat, Sdjule, im iffentlichen wie im Prinatleben fich manifeftiten fold. Der Begriff Des Red)ts und Der Begriff Des Guten find untrennbar. Diejer betrifft die

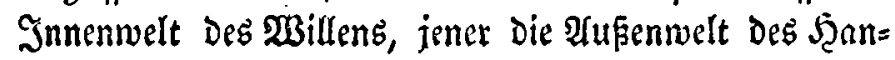
Delns, uno beide fteben unter einer uno Der námlichen Soee Der menjalichen Perjonlichteit, in Der fich Dab Unfidtbare verfiatbaret, und Das Sidtbare auf Das


Melt Des Guten - in uno mit Dem Menjhen ju= gleid) in Die Erfocinung tritt: fo tritt aud zugleids in Dieje şirflíffeit mit Der Geburt, ja yor Der Beburt

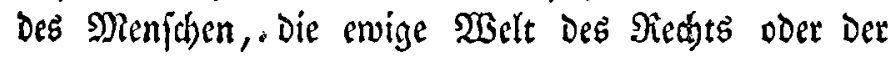
Staat. Sied)t und Moral bieten, cinandet wie zwei ungetrennte und untrennbate $\mathfrak{B e g r i f f e}$ die Şand. Das Bermunftred)t ift die áupere Seite, gleidjfam die (Er= itheimungewelt Der Moral in Dem Reben gegenjeitiger. Beziclungen Der Menjchen, ja in Der Bezieljung alles Intelligiblen auf wirflithe und mógliche Şandlungen. 



wohl fü Die innere nle iufere $\mathfrak{B}_{\mathrm{C}} \mathrm{lt}$, Dort Der $\mathfrak{B} e=$ grif, Dns benuftefein der moralifben Nóthigung,

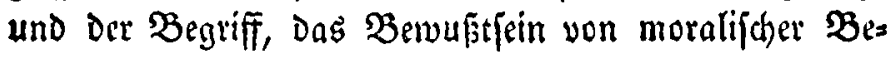
lohnung und Strafe; und bier, in Der ápern Splyare Der Mianifeftation, Der Begriff Des Red) $t \mathfrak{s} \mathfrak{w}$ anges und Der aus dempelben fich ergebenden $\Re$ ed) ts ft $r$ afe. Sif nun der Red)tšbegriff Der Strafe felbfit ein $\mathfrak{B e r}=$ nunftbegriff, ein Begriff Der Freilyeit und Des yon Der= felben abliángenden Rechtşzonges: fo benegt uno ftükst (ii) aud) ber $\mathfrak{B}$ egriff yon Strafe auf Das (jpbiet oder Den $\mathfrak{B e g r i f f}$ der Freibeit. Wie die moralifdhe Niothi= gung Der Freilyeit die $\mathfrak{B e r}\{$ d)uldung Des $\mathfrak{M e n}[$ (h)en zur Etrafe zielt und diefe ßerfduldoung Durdh die Strafe Des Seniffens und Des $\mathfrak{B e n u f f t f e i n s ~ r i d ) t e t : ~ f o ~ i f t ~ a u d ) ~}$ Der $\mathfrak{B} e g r i f f$ und Das (sebiet Des Siecl)tszmangrs unmit= telbar der Şnhaber uno Sichter ủber die zu verfúgende und zu vollftrecfende Sied)teftrafe, Die als foldbe von

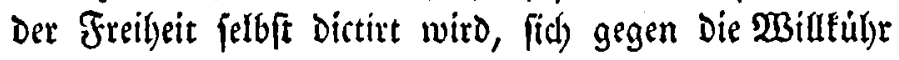
Der Redtsverleşungen wendet, wo aljo die Freitheit felbjt den Menfden ftraft, indem fie die Mitufübr unter Das loblere Gejeß̧ Der Freilyeit als eines Red)tşuan= geb ftellt. Es ift in hódffen Grade widerjpredhend, Dem Beferze Der Bernunftfreibeit gerndegu entgegenges

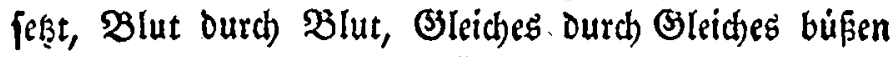
und yerjolynen zu wollen. Es beißst, „Der Mórder habe ,fich felloft Des Bied)tes jeiner Perjonlid) feit begebert, uno „és gefdelye ilym fein Unred)t, wenn ex am Seben ges "ftraft werde." Ilber wie fann fid ein Menf(h) eines uriprungliden und notljwendigen Sechts begeben? auf 
welde $\mathfrak{B}$ eife, wie Ennn bie Perponlidfetet vertilgt und aus Dem Reidse der Beifter ausgeftriden werden? Urs fprünglidhe Rechte Der Menjabeit find ewig, unvertilgs bar; und wenn aud Sd)atten, Finfterniffe über Die Sonne lingelyen, fo bleibt Dodh immer diefes frrablende Ridyt, die Sonne Der Sdhopfung. Und aud jugeges ben, Der Mórder mache jich burch feine Unthat dez Rech)ts Der Perfónlichfeit yerluftig, folgt Daraus für Den

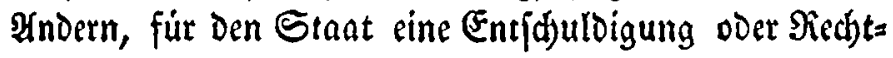
fertigung, Den $\mathfrak{B e r b i e ( b e r ~ a u d ) ~ n a d ) ~ D e m ~ B e r l u f t e ~ D i e = ~}$ fer Perjónlid)feit ju meffen? ift fidh Der Stant nidjt aud) feine eigne Perfonlichteit, feine eigne ad)tung vor

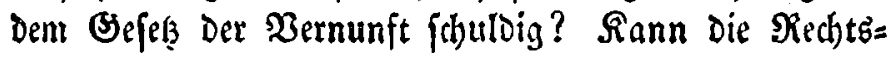
verleşung, die Miffethat Dem Ridhterjpruche ein Redst geben, Das Giejes Der Perfönlichfeit nicht ju ad)ten

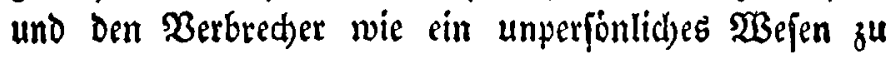
bandhaben? Sann und Darf, fragen wir, wenn und wo nur irgend Moral gilt, Bojes durd) 2 ójes vergol= ten und augegliden werden? (Fo berrid)t ja felbfr

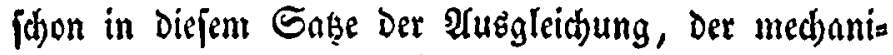


Wider[prudb. Wie Eann eine Rechteverleķung Dadurd) gut gemad)t werden, Daf wieder irgend eim Recht ver= fưmmert, ein शiecht zur Strafe gezogen wird. Wenn felbft fobn in Dem gewolinlid)en Berfelyr des menfche lichen Rebens unter Den Sadjen ein fo groper Unters fdied berridt, Daß nidt alles nach bemjelben Preife, nach Demjelben Pfund und (Sewidt umgened)jelt wer: Den fann, fondern Der innere uno aufere zufállige Wettl) Der Dinge in $\mathcal{A}$ (j) hlag genommen werden mus: 
fo Findet ja lier ein folấer linter(d)ied, Der burch nid)t

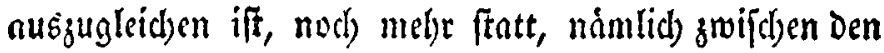

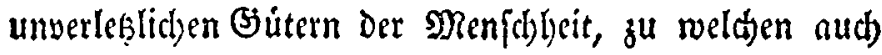
Die leiblidye Exifteng Der Men[d)ennatur geljort, und Denjenigen Eủtern, Die nur einen àuseren, bedingten


Die gróste Miffetlat ift, wsil er fich ummittelbar an Der moralijden, notlwendigen Exiftenz oder Erijdeinung Der Menjdhennatur vergreift: fo fehen wir nidbt ab, Durd) weld)en Suftigact, Duttd) weldben Begrifi Der Gea red)tigfeit das Şochgerid)t, unter weldbem die Exiiftenz Des $\mathfrak{B e r b r e d b e r s ~ f a ́ l l t , ~ u n d ~ w o d u r d ) ~ e i n ~ u r i p r u ́ n g l i t b e s ~}$

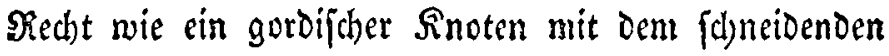
Sd)wetote Durdbauten wird, vertheidige uno entidjul= Digt werden fann. Der Begrifi Ler Gered)tigkeit ift nid)t Denfbar ohne Begieflung auf Die Yyflid)t Der $\mathfrak{A} d$ )= tung gegen fith jellbit und Den Mlad)ften. Der intelli=


auf Die Beflandlung Der Menfdennatur pover Der menf(d)= lidhen Eviftenz ift und bleibt, bie Perjönlid)feit mad)

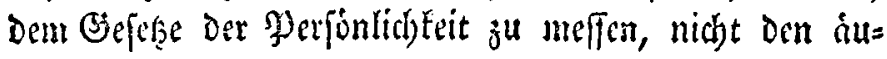

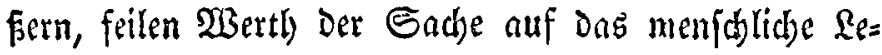
ben anjuwenden, fondern wo es Strafe giebt und gilt, nuch Diefe Strafe mit Den Beferezen Der menfdjliden, perfönlithen Freitheit úbereinftimmen zu laffer. Der fo

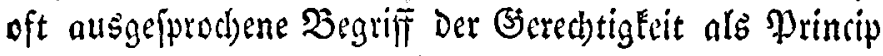
und Eeele Des Etants if und Darf nidht jene rolje sibiedervergeltung fein, Eache um Sadje zu ơpfern, fonbern er iff Die böbere Freilgeit uno Madjt Der $\mathfrak{B} e r=$ nunft, die $\mathfrak{P e r j o ̈ n l i d f f e i t ~ z u ~ a d d e n , ~ G d j u l d ~ u n d ~} \mathfrak{D} e r=$ 
bred)en nur an Denjenigen Butern Des Lebens zur Strafe zu zielden, Durdb weldbe Das Befüll, das $\mathfrak{B e}=$ wufitjein Der Perfonlidfeit, Des lobkeren Menjolyen= werths erbalten uno befeftigt wiro. Es fino fold)e Giter, bie, un die menjoblidbe Freilyeit fu erweifen, Durd) die Freil)eit felbfit erzeuge und geboren werden.

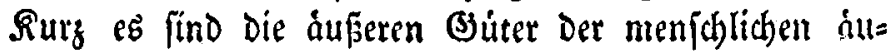


Bell u. f. w., Dies find Die Sbjecte und $\mathfrak{D}$ ittel, Durd) welche und an welchen Durd) Den Redbtejnang Der Etrafe Der Menfdh feine Red)tรverlesungen büßen uno für diejelbe den nothwendigen Erfạ geben Eann. So wird die freie $\mathfrak{W}$ clt Des Rechts Durd) Den Rechtsjmang,

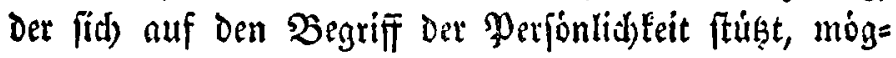
lid) uno notlunendig; Die Strafe ift Dann nicht ein

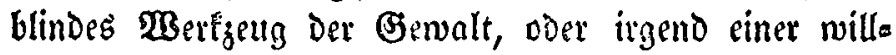

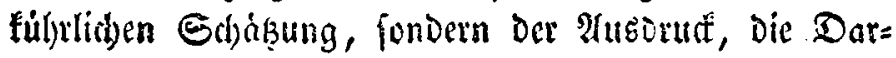
fteflung Der Miad)t uno Freilgeit Der Sernumft, inders fie Den Menjthen aus Der Naturgewalt zu einem freien Reidbe geiftiger, moralif(her Bergeltung erlyst. Das Etrafred)t wiro in einem entigen Conficte mit fith felbit bleiben, fo lange es Bergeten uno Strafe nod) áuseren Suantititen uno Sualitaten mifit, wo fid die willfúbrlichfte, zufálligfte Srala ergicbt, bis zu


bred)en gealundet werden finmen. Der Begriff Der Red)tsftrafe fúl)ut ein intelligibles Moment in uno mit


nad) ihren blos autsern Erfolgen, fondern, in wiefern fie zur Strafe gezogen werden, nadj Der unemolidben 
Grópe, welche Der Begriff Der Fteibeit nit fich fülst,


Der Strafbeftimmung zu Stonde fommt, als nad) Der blos medhanifichen Seftimmung. Uno es ift Dalyer aud)

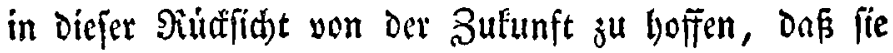
mildere, gerechtere Strafgefe(ze einfülyren werbe, daß Bergelen und Berbred)en nidht nad) Zahlen yon etwa funfzig Stoúf(b)lígen bejallit, fondern nadl) Dem Man $\tilde{\beta}=$

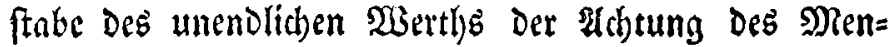


Gnren (Bütern Der Menjhenwürde ermefien und vergol= ten werden. SBir haben uber diejen Gegenftand un= fere Heberzeugung mehrfach in Den Abljandungen åber Dne Strafred)t ausgefprod)en und find bei Der Dat= ftellung unjeres Bebanten eines gewiffen zu lebendigen

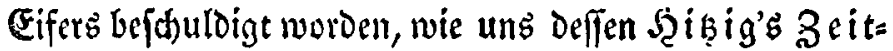
fdrift úber Die Eriminal = Red)tepflege 1833 1. Band anflagt. $\mathfrak{A}$ ber wir geben ju bedenten, Dns dieje $\mathfrak{s e b e n d i g f e i t ~ D e r ~} \mathfrak{D a r f t e l l u n g}$ nur entjprang aus jenen bittrin $\mathfrak{B o r w}$ infen eines uns gegenúberftelyenden Mufticismus; wit machten uns námlid) Durd) Die $\mathfrak{\text { (tht }}$ uno Weife, wie wil bie memidhlidbe Natur betrad)te= ten, Diefe nicl)t als cin Erbbojes, als cine Fortpflan= zung oer Erbfünde, fondern als sin Erbtheil yon man= nigfaltiget Ed)wadbe uno (jebredblid)feit, als eine (Frs f(b)inumg felly beoingter uno bedingender Srganifatio= nen alzujelen, — in Diefer J̧injicht Der tadelfafteften, undififtid)er (befinnungen fiduloig. Bon diejen

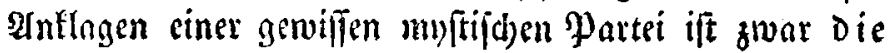
Criminal= Rechtopplicge, die nad) Dem Budbftaven ge= 



pfiege vergel)t fid) wieder allf eitre andere Prt gegen bie aufflárenden Fortidyritte Der Wiffenfdaft, indem fie nach Dem Terrorismus einmal cingefülyter und yer= vroneter $\mathfrak{B e f t i m m u n g e n , ~ n i d ) t ~ a b e r ~ n a d ) ~ g r o ́ p e c e r e r ~ u n d ~}$

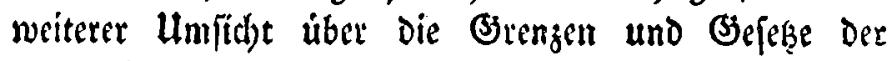
menjd)lichen Patur, Das Urtheil úber. Jutechnung uno Strafe fállt. $\mathfrak{W}_{\mathrm{er}}$ trágt nidjt nod) Die rigorifti(d)en

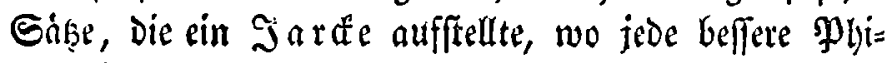
lojophie in Den Bann juridifder Sentenzen gelegt wurde, leider nod) in feinem (Sedadhtnif!

Unter die Paradoçieen Des menflylicben Betftan= Dis geljort nuch, Daß man yon der moralifiden uno religiojen Seite Das Recl)t, die Befugnif Der Todes= frafe Durch einen alten mojaifden 2 (usjprudh bat auf= recht exhalten und fonctioniren wollen, alò wenn bie Buidjer des neuen Bumbes, Der d)riftich philofophi= fhen Sel)re nicht andere, yollfommmere Ifusfprúche ent= bielten. Ueber die Art Der Snterpretation fann man zwar Mnannigfaltig freiten, und es crgeben fid) bier verjchiedene, ja ganj entgegengefeşte Meinungen, ic nad)oem man sinen blos literalen, oder aud biftori= (d)en, oder endlich moralijhen und philofoplifichen Sie=

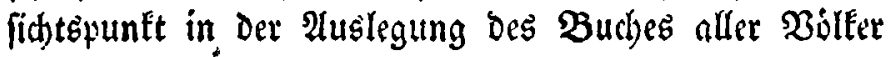
wiblt. Die Jurdyt ift Der Infang Der Şirrardfie, jo= wohl Des̀ geiftlichen als weltlidben Stubls. Die Sirche weip (idh anfangs nicht anders zu ftüisen, als burd) fiteng und eigenliebig vorgejdriebene Gebote. Und Der geichwinde, zudedende Bannftrahl der Strafe ift ber

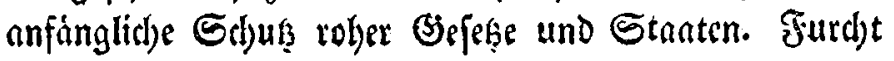


und Desputie, Glinder Fifer und Şierarbie find die erften Sinder Der Sindheit. Der mufaifde $\mathfrak{\text { Teeres } =}$ zug, ber, wie eine bomerifahe Sliade, fïh) in Dab ge= lobte sano gen Serufalem wanite, fonnte nid)t an= Ders, als nit Der Flammenfiule Der áuferen Genalt, fiic) Den $\mathfrak{B e g}$ Durd) die Wuiften, Durd) Die sinóden ju ferneren Salbrlunderten eoleter 2lufflárung balnen. WBas anfangs mit Den Flammenzügen Des Zoins, Des Blif̧es gejádrieben war, erlellte fich) fpáter unter Der Margenróthe einer reineren $\mathfrak{B o l l e n d u n g . ~} \mathfrak{B}$ er unbe= fangen fich Dem Geifte, Der die driftliche Religion, die Buider und $\mathfrak{B e r b e i} \beta$ ungen des neuen $\mathfrak{B}$ undes be= feelt, hingiebt, wird nidht anders, als eben fo unbefan= gen nad) Dem Grifte Diejer Bruberreligion wever in weltlid)en nod) geiftlid)en Dingen Den Strb bredsen




Der $=$ ooer Níd)iten $=2 i e b e$ wird bier Den janften Stab Der Duldung, Der Befferung, Der Ergielung wáblen, weil folde Strafe, die youm Şimmel fommt, meht und coler wirft, als Die Steinigung jones armen $\mathfrak{W}$ e $=$ bes, die am Gabbath Şolz aufgelefen hatte, und weil joldse Etrajen Der $\mathfrak{B e f f e r u n g}$ und $\mathfrak{B}$ efreiung mely mit Dem Beifte Der Religion, Der Mornl und Plilojowlye übrreinftimmen. Die Moral, das Chriftenthum ges bietet $\mathscr{U}(\mathfrak{b})$ ung und liebe you Dem Merifhenred(f)t. Und wie Eann alfo bei Diefem Gebote, bei Diejer Menjalen= wưbe, olne weldbe Erin $\mathfrak{B u n d}$ auf (Erden beftellet, Der Faden Des menidhlidsen Dafains Durd) einen welts lid)en Ridjter zerrifijen werden, cin Faden, Der fita) fo 
furg, fo Iang Durdis fo mannigfaltige Beridslingungen Des innern und áußern Lebens, yon ier $\mathfrak{W i e g e ~ b i s ~}$ zum Grabe, yon Dem leben Des Fótus im Mutter= (d)oofie bis zu jener alleinigen 2luferfteflung binfdlingt. Das menichliche seben ift in fich ein fo fartes Se= webe, fo mannigfaltig verwicfelt und ourdfflod)ten, DaF man fid) wobl húten mag, das (Sewebe nod) melyr fu zerreipen, in weldyes fid die Sturme gelegt lyaben; wir migen Die Yjinde, dieje fo unbefannte, unfidtbare Macht, wolyl ju fobisen und ju waliren fucben, felbft

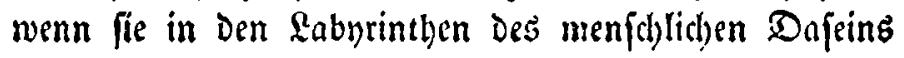
Ungebeuern yon Reidenjd)aft, yon Unwiffenlyeit, yon Bedantenlofigfeit, yon unglưflichen Ergebniffen inne= rer und áfierer Bedingtheit melse oder weniger fur Beute geworden ift. Bas fid úber eine folche mo= ralijd)e voer religiópe $\mathfrak{A}$ nifitht in Begiefung auf bie $\mathfrak{a b}=$

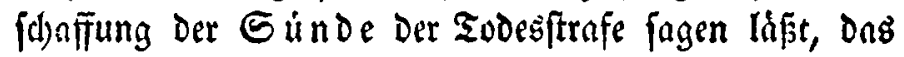
ift yortrefflich entwicfelt in oer folgends mitgetheilten Sil) leiermad)eriden Predigt, die mit fo vieler Umfid)t, mit fo vieler Wribe der §raft Den Feuereifer theologifd)er anderer Befenntniffe zì befänftigen und fu befd)widftigen fdjeint. (Extrubt mag es fain, hier die brieflid)en Worte eines Dem großjen $\mathfrak{H}$ einigegange= nen nabe befreundeten Mannes, Der felbft an ber J̧er= nusgabe folgẻnder $\mathfrak{B l a t t e r ~ D e n ~ t h a ́ t i g f t e n ~} \mathfrak{A}$ ntheil nimıt, als eine J̦offnung und $\mathfrak{B e r h e i p u n g ~ b e r ~ g u t e n ~ G a d b e ~}$ beizufügen. "(E) mag vielleid)t einem jüngeren (Se: "idbled)te yergoint fein, Den hellen $\mathfrak{F} a g$ einer Bufunft "gu fdauen, Deren Morgenrutle wir nut alynden, uno




"und Riebe begrunden wird, in welchem ftatt des tod= "ten Budsftaben Der frei gewordene Beift, und ftatt „einer leeren, aber nbguttijd) vereljrten Form ein fitt= "lich uno driftlidh reiner Sinn lerriden wiro, Der, "ftatt Den Mitbruder zu fränfen uno zu vernichten, „ilhn fu erheben und zu beglüfen tradten wird, wie "es in Gottes beiligem $\mathfrak{W}$ Sillen liegt, uno wie es bie


"fichen fordern. $\mathfrak{D a}$ aber Sottes $\mathfrak{B}$ Bille dod einmal "Erfüllung finden mus, fo ift das $\mathfrak{B}$ ild einer alfo "veredelten Bufunft mel) als ein Traum oder als Das "₹rugbilo einer úber\{pannten Plyantafie." -

Was bie antlyropologifd)en Erunde belangt, welde für die nothwendige 2 uflebung der Todesftrafe fpre= d)en, fo ubergelyen wir lier biefe, da fie fo oft fison Der Gegenftand unjerer in mełreren Beitjdriften abge= Dructen Unterfuchungen gewejen find. Sin eimer fole Wen Unterjudbung thaten wir die Anfrage, ob es nidht wenigltens verfudboweife rathjam fei, auf einige Seit Die Todesftrafe zu jugpendiren, . um dann viefletht ein Defto unparteificheres, empirifd)es Urtheil úber die Noth)= wenoigteit oder (Entbefyrlidfeit jener Blutwerldingniffe fállen zu fónnen. $\mathfrak{A} b e r$ es fibeint, daß unjer Bcitalter auch nicht einmal dieje innere Sraft úber fich gewin= nen fann, simen folchen $\mathfrak{B e r j u c h}$ nnfuftellen, ob es fdjon immer und ftets bei feinem beliebten Sprúd)wort yon Exenupel ftatuiren bleibt. (Fin foldher Derfud) wưrde freilid aud, in wiefern auf Demfelben die bódbfte und leşte (Entf(d)eioung beruben follte, felye mißlid) uno sweifellaft fein; Denn Das Redist und Unred)t fann 
nidit Durd) Experinente erfunden und erprobt werden;


Heber Dieje anthropologijhen Gründe, weldhe Die $\mathfrak{A b}=$ fhaffung der Sodesftrnfe amratben, find viele Mate= rialien yorbanden, jin fie find felbft foon melyr oder

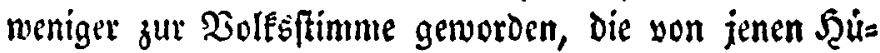
geln ber blutigen (Sered)rigfeit nad) milderen und ebles ren $\mathfrak{B}$ eftinimungen Der Strafe ruft.

Folgende Blàtter enthalten die Mittbeilung Iand: ftándifher Berlyandlungen fúr oder wiber die mógliche Erfüllung unferer eingereichten Bitte uno Petition, Denfnuidoige Sd)riften verdienftyoller Minnner für ober wider das Recht der Todesjtinfe. Dieje Mittheilun= gen des grófeften Interefles werden ergånzen, was meinen eigenen Bemerfungen und Einwendungen an Siraft, $\mathfrak{D o l l f}$ tándigfeit und Flarerer Entwiffelung $a b=$

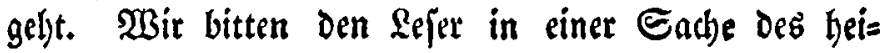

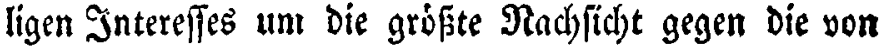
uns begangenen Feller uno Itrthümer, aber aud) um Wabrheitgliebe und Diejenige Freimúthigfeit, weldhe Den Tndel bei aller Sdbonung ernft und obne Rưf:= ficht ausfpricht. Wir glauben Durd) die Sammlung folgender Sdriften Dem yaterlándijchen und Dem all= gemeinen menichlithen Jutereffe einigen - und viels leid)t Den leşen Dienft geleiftet zu Gaben. Denn wer cine längere Seit feines Rebens nicht ohme mannigfals tige Opfer Des Gemuths uno Eeiftes fich Der Sojung und Beantwortung verwifelter irdifher Probleme lyin= gegeben hat, felint fid) ja endlich wohl, inden er bas Sagewert ruhig úberifluat, nadh einer sojung bes 
gróften uno beiligiten Problems, weldyes auf die


Difhe mit Dem Evigen yerbindet und úber Dem Sir= Difden Die Morgenróthe eines befferen Tages aufbre=

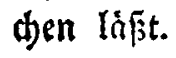

Drebden, im December 1834.

Der $\mathfrak{B e r f a f i e r . ~}$ 